Please do not remove this page

RMIT

UNIVERSITY

\title{
An improved visual pruning algorithm for perceptually lossless medical image coding
}

Wu, David; Tan, Damian; Wu, Hong Ren

https://researchrepository.rmit.edu.au/esploro/outputs/9921859601801341/filesAndLinks?institution=61RMIT_INST\&index=null

Wu, D., Tan, D., \& Wu, H. R. (2005). An improved visual pruning algorithm for perceptually lossless medical image coding. Proceedings of the TENCON 2005 IEEE Region 10 Conference, 794-799.

https://doi.org/10.1109/TENCON.2005.301333

Published Version: https://doi.org/10.1109/TENCON.2005.301333

Repository homepage: https://researchrepository.rmit.edu.au

(c) 2005 IEEE. Personal use of this material is permitted. However, permission to reprint/republish this material for advertising or promotional purposes or for creating new collective works for resale or redistribution to servers or lists, or to reuse any copyrighted component of this work in other works must be obtained from the IEEE.

Downloaded On 2023/04/26 18:13:25 +1000 


\title{
An Improved Visual Pruning Algorithm for Perceptually Lossless Medical Image Coding
}

\author{
David Wu, Damian M. Tan and Hong Ren Wu \\ School of Electrical and Computer Engineering, \\ Royal Melbourne Institute of Technology, \\ Melbourne, Victoria, Australia, 3001 \\ Email: Henry.Wu@rmit.edu.au
}

\begin{abstract}
An improved algorithm for perceptually lossless coding of medical images is presented in this paper. Built on the JPEG 2000 coding framework, the proposed coder combines an improved Visual Pruning algorithm with an advanced model of the Human Visual System to identify and to remove visually insignificant/irrelevant information. Current results have shown superior compression ratio gains over that of its lossless counterparts without any loss in visual fidelity.
\end{abstract}

\section{INTRODUCTION}

Advanced medical imaging systems play an important role in providing a non-invasive, fast and accurate diagnosis. The key to this is through the digitisation of medical images. Digital medical images can be stored indefinitely without any deterioration in quality and can be transmitted to any geographical location with relative ease. One type of medical application that utilizes these features is telemedicine [1]. However, as the demand increases, so does the requirements for storage space and network transmission bandwidth. Thus, the challenge is how to deliver clinically critical information in a smaller package. A solution to this problem is through image compression. In general, there are two broad categories for encoding digital images; they are reversible and irreversible coding. Reversible coding schemes offer the advantage of having no loss of information. Although desirable, the current state-of-the-art reversible image coding schemes do not provide adequate compression ratio gains for applications in medical imaging [2]. Counter to this is irreversible coding schemes, which provide greater compression ratio gains at the expense of information integrity. However, a distinction of what information is lost must be ascertained. Past publications have shown that compression ratios up to 1:20 are achievable without any loss in diagnostic information [2]-[6].

An alternative to these schemes is perceptually lossless image coding (PLIC). PLIC provides the best of both worlds, that is, greater compression ratio gain than reversible coding schemes, while producing images without any visible loss. The effectiveness of this concept was demonstrated in [7], with 27 radiologists and 4 radiographers. This paper presents a novel algorithm for visual pruning (VP). Built on the JPEG 2000 coding framework [8], the algorithm is combined with an advanced model of the Human Visual System (HVS) [9] so that only visually irrelevant/insignificant information is removed. Thus having the ability to encode images at a perceptually lossless quality. Other key features of this algorithm are its simplicity and modularity. These features enable the algorithm to be implemented into any other Wavelet transform based coding framework without disrupting bit-stream compliance. The effectivness of modularity has been demonstrated in [10], [11] with a different algorithm using the Set Partitioning in Hierarchical Trees (SPIHT) coding framework [12].

This paper is presented with the following sections. Section II describes the vision model employed in the improved VP algorithm. Section III, describes the VP algorithm as well as its adaptation into the JPEG 2000 coding framework. Section IV evaluates the improved VP algorithm and finally a conclusion in Section V.

\section{The Perceptual Distortion Metric}

Traditional raw mathematical objective metrics such as Mean squared error (MSE) and Peak-signal-to-noise ratio (PSNR) has served as one of the basic means for quantifying visual distortions/quality [13]. However, the main drawback of these traditional metrics is that they do not correlate with what is perceived by a human observer [14]. Thus, the need for metrics that incorporate the perceptual characteristics of the HVS, that is, a perceptual distortion metric. The effectiveness of these HVS based metrics have been demonstrated in past publications [15]-[18]. This section describes a perceptual distortion metric embedded with a vision model, however, development and fundamental ideas are left the references [9], [16], [18], [19].

The vision model [9] in this paper is based on the unified vision model template, the contrast gain control (CGC) (Figure 1), by Watson and Solomon [18]. The CGC consists of three parts and takes in two inputs, a processed image and a reference image, both of which are subjected to the three part process. The three parts of the CGC are linear transform, visual masking response and detection and pooling. A linear transform (Equation 1) takes into account of the frequency and orientation selectivity of the HVS [18], [20]. In general, a linear transform can be expressed as

$$
\mathbf{X}=T(\mathbf{x})
$$

where, $\mathbf{X}$ and $\mathbf{x}$ represents the neural and pixel domain images, respectively. Immediately following the linear transform, a set of contrast sensitive weights are applied to modulate the 


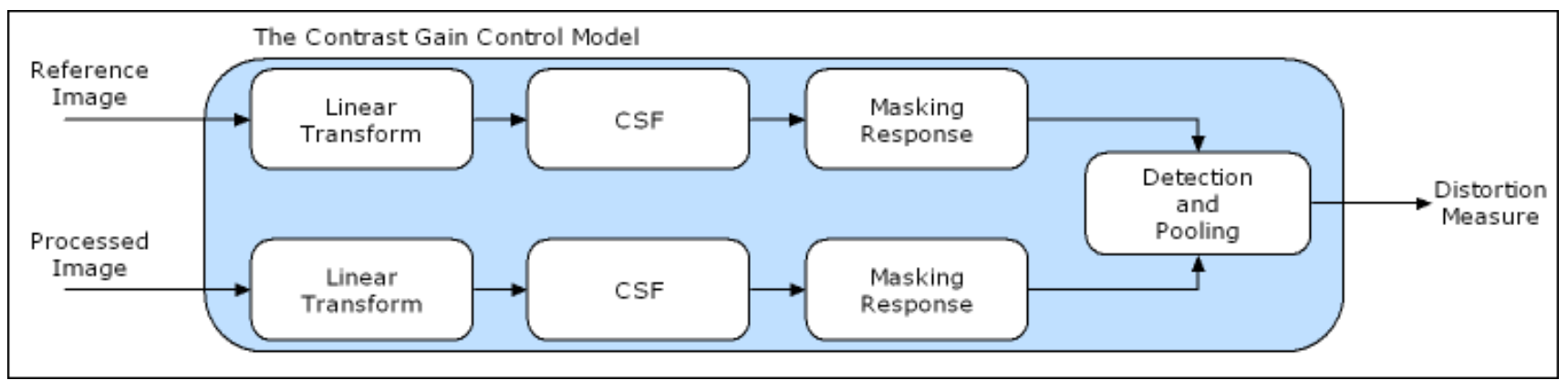

Fig. 1. The Contrast Gain Control Model. The example given here only models the primary visual cortex. The CSF can be applied prior to or after the frequency decomposition. The difference between the two approaches is the domain in which the CSF operates in - time or frequency. Here, the CSF operates in the frequency domain.

neural image to the sensitivity levels of the human eye. The selection of a transform is an issue. Generally over-complete transforms, such as the Steerable-Pyramid [21] (SPT), are used since they can closely represent the mechanics of the HVS [20]. However, although over-complete transforms, like the SPT, are 'alias-free'; they require additional resources to code. Thus to counter this problem, a Mallat [22] Wavelet transform with the Daubechies 9/7 filter set [23] (D97) is used. Only 5 levels of decomposition was used due to the sensitivity of the vision model ${ }^{1}$. The drawback of using the Wavelet transform with the D97 filters is contrary to using over-complete transforms [20]. Despite these issues, using the 5 level Mallat [22] Wavelet transform opens up practical advantages such as bitstream compliance with JPEG2000 [24], which subsequently conforms with the Digital Imaging and Communications in Medicine (DICOM) standard [25].

Visual masking causes a visual signal to be hidden or diminished within the presence of another visual signal. This occurs between neurons from similar (intra-) and different (inter-) frequency, orientation and colour channels ${ }^{2}$. It is these interactions that are modelled (Equation 2).

$$
R_{\mathcal{Z}, l, \theta}[m, n]=k_{\mathcal{Z}} \cdot \frac{E_{\mathcal{Z}, l, \theta}[m, n]}{I_{\mathcal{Z}, l, \theta}[m, n]+\gamma_{\mathcal{Z}}^{q}},
$$

where $m$ and $n$ are the spatial frequency coordinate of a coefficient, $E_{\mathcal{Z}, l, \theta}[m, n]$ and $I_{\mathcal{Z}, l, \theta}[m, n]$ are excitation and inhibition functions, $k_{\mathcal{Z}}$ and $\gamma_{\mathcal{Z}}^{q}$ are the scaling and saturation constants, $\mathcal{Z} \in\{\Theta, \Upsilon\}$, with $\Theta$ and $\Upsilon$ specifying the inter-orientation and intra-frequency masking domains, respectively $^{3} \cdot l=\{1,2,3,4,5\}$ and $\theta=\{1,2,3\}$ represent the frequency levels and the orientation bands, respectively. The excitation and inhibition functions for each domain are defined as follows:

$$
\begin{aligned}
E_{\Theta, l, \theta}[m, n] & =\mathbf{X}_{l, \theta}[m, n]^{p_{\Theta}} \\
E_{\Upsilon, l, \theta}[m, n] & =\mathbf{X}_{l, \theta}[m, n]^{p_{\Upsilon}}
\end{aligned}
$$

\footnotetext{
${ }^{1}$ Sensitivity here refers to the vision model being calibrated to only a 5-level Wavelet transform.

${ }^{2}$ Colour masking is not considered. This paper focuses on grayscale images.

${ }^{3}$ Inter-frequency masking was omitted to simplify the model.
}

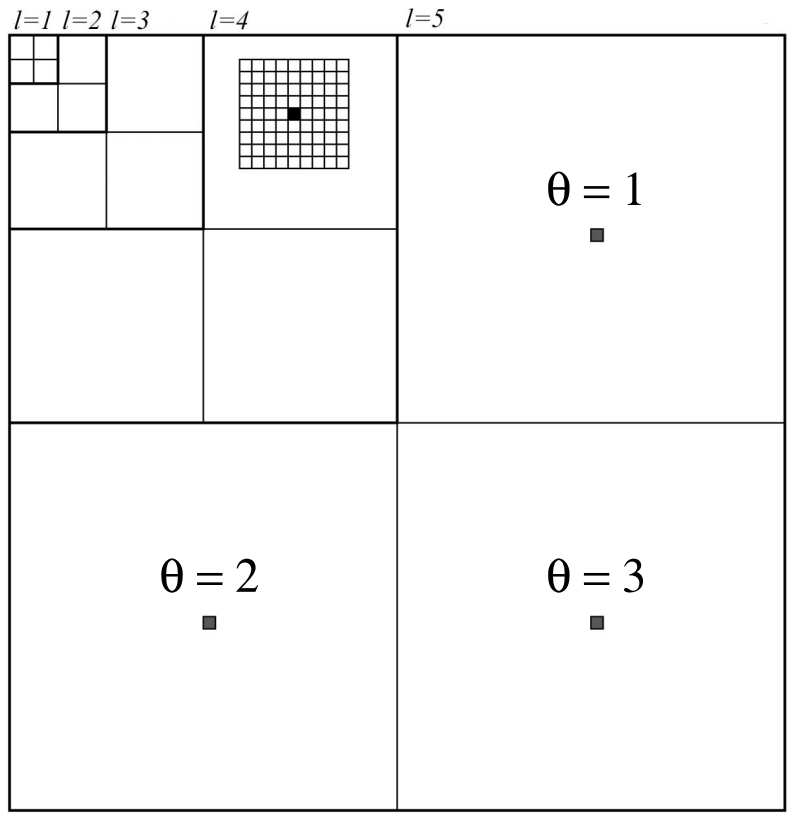

Fig. 2. Orientation and spatial frequency locations of the hierarchical (Mallat wavelet) decomposition. Each frequency level has three orientated bands, $\theta=$ $\{1,2,3\}$, except for the lowest frequency level. At the lowest frequency, there is an additional isotropic band (LL) - top left corner. At frequency level 4, the centre (shaded) coefficient represents $X_{4,1}[m, n]$ and the surrounding coefficients are $X_{4,1}[u, v]$ with $u=\{m-4, m-3, \ldots, m+3, m+4\}$ and $v=\{n$ $4, n-3, \ldots, n+3, n+4\}$.

$$
\begin{aligned}
I_{\Theta, l, \theta}[m, n] & =\mathbf{X}_{l, \theta}[m, n]^{q}+\sum_{\alpha=1, \alpha \neq \theta}^{3} \mathbf{X}_{l, \alpha}[m, n]^{q} \\
I_{\Upsilon, l, \theta}[m, n] & =\frac{8}{A_{l}} \sum_{u=m-l}^{m+l} \sum_{v=n-l}^{n+l} \mathbf{X}_{l, \theta}[u, v]+\sigma_{\text {var }_{l}}^{q}
\end{aligned}
$$

where $\mathbf{X}_{l, \theta}[m, n]$ is the transform coefficient at orientation $\theta$, spatial frequency location $[m, n]$ and frequency level $l$. (See Figure 2). $I_{\Theta, l, \theta}[m, n]$ is the sum of transformed coefficients spanning all orientations. $I_{\Upsilon, l, \theta}[m, n]$ is the sum of neighbouring coefficients about $\mathbf{X}_{l, \theta}[m, n]$ (Figure 2). The neighbourhood, $A_{l}=(2 l+1)^{2}$, is a square area surrounding $\mathbf{X}_{l, \theta}[m, n]$, whose size is dependant on the frequency level of $\mathbf{X}_{l, \theta}[m, n]$. Thus, coefficients from the highest fre- 
quency level would have the largest neighbourhood. This approach attempts to equalize the uneven spatial coverage between images of different frequency levels inherent in multi-resolution representations. The neighbourhood variance $\sigma_{v_{\text {ar } r_{l}}}=\frac{1}{A_{l}} \sum_{u=m-l}^{m+l} \sum_{v=n-l}^{n+l}\left(\mathbf{X}_{l, \theta}[u, v]-\mu\right)^{2}$, with $\mu$ representing the mean, has been added to the inhibition process to account for texture masking [26]. Exponents $p_{\mathcal{Z}}$ and $q$ are governed by the condition $p_{\mathcal{Z}}>q>0$ according to [18]. Currently, $q$ is set to 2 .

The final component of the model detects the perceptually significant difference between two images. A squared-error $\left(l_{2}\right.$ norm) function defines the distortion within each masking channel. The total distortion is the sum of the distortions over all masking channels, given as

$$
D_{T}=\sum_{l=1}^{l_{\max }} \sum_{m=1}^{M_{l}} \sum_{n=1}^{N_{l}} \sum_{\theta=1}^{3} \sum_{\mathcal{Z}} g_{\mathcal{Z}} \cdot\left|R_{a_{\mathcal{Z}, l, \theta}}[m, n]-R_{b_{\mathcal{Z}, l, \theta}}[m, n]\right|^{2}
$$

where $R_{a \mathcal{Z}, l, \theta}$ and $R_{b_{\mathcal{Z}, l, \theta}}$ are the masking responses of the two images, $a$ and $b$, respectively, and $g_{\mathcal{Z}}$ being the channel gain. $l_{\max }$ is the maximum number of decompositions and $\mathcal{Z} \in\{\Theta, \Upsilon\}$, where $\Theta$ and $\Upsilon$ are the inter-orientation and intra-frequency masking domains. In this paper, $l_{\max }$ is set to 5. Given that the dimensions of the image are $M \times N$, $M_{l}$ and $N_{l}$ are defined as $M_{l}=M \times 2^{l-L}$ and $N_{l}=N \times$ $2^{l-L}$, respectively, where $L=l_{\max }+1$. The pooling equation, Equation (7), pools all coefficients spanning all frequencies and orientations, and provides an overall perceptual distortion, $D_{T}$ between the two images.

\section{VISUAL PRUNING}

\section{A. The Proposed Algorithm}

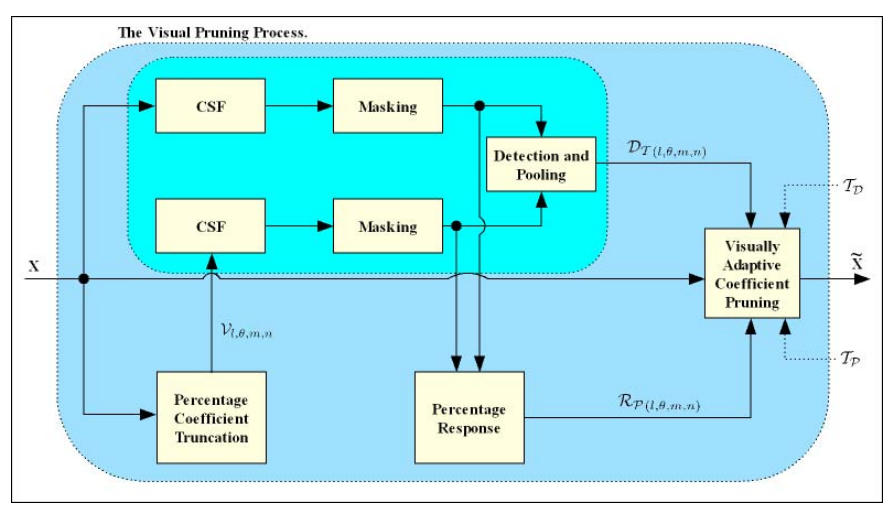

Fig. 3. The Visual Pruning Algorithm.

By employing the perceptual distortion metric described in Section II, the VP algorithm (Figure 3) can identify and remove visually irrelevant/insignificant information. For a given frequency level, $l$, orientation band, $\theta$, a spatial frequency location $(m, n)$ and $\mathcal{B} \in\{0,1, \ldots, P\}$, the $\mathrm{VP}$ algorithm can be described in two stages. The first stage computes a set of distortion measures (Equation 7), $\mathcal{D}_{\mathcal{T}(l, \theta, m, n)}=$ $\left\{D_{T_{i}(l, \theta, m, n)} \mid i \in \mathcal{B}\right\}$, and a set of percentage responses, $\mathcal{R}_{\mathcal{P}(l, \theta, m, n)}=\left\{R_{P_{i}(l, \theta, m, n)} \mid i \in \mathcal{B}\right\}$, from a reference image and a set of processed images, $\mathcal{V}_{l, \theta, m, n} . P$ is positive nonzero value specifying the precision of truncation. Larger values of $P$ will result in finer truncated coefficients and hence provide a more accurate account of the visual distortions. The processed images are generated by percentage coefficient truncation (PCT)

$$
\tilde{\mathbf{X}}_{i_{l, \theta}}[m, n]=\left\{\mathbf{X}_{l, \theta}[m, n] \times \frac{(P-i)}{P} \mid i \in \mathcal{B}\right\}
$$

where, $\tilde{\mathbf{X}}_{i_{l, \theta}}[m, n]$ and $\mathbf{X}_{l, \theta}[m, n]$ are the processed and original coefficients, respectively. Large values of $\mathrm{P}$ are desirable at this stage since visual distortions can be accurately modeled. $P$ is set to 100 in this paper. Equation 8 is applied to each coefficient separately at each frequency band except the LL band. The percentage response, $R_{P_{i}(l, \theta, m, n)}$, for a given reference coefficient and a distorted coefficient, is defined as

$$
R_{P_{i}(l, \theta, m, n)}=\frac{\sum_{\mathcal{Z}} R_{i_{(\mathcal{Z}, l, \theta)}}[m, n]}{\sum_{\mathcal{Z}} R_{O_{(\mathcal{Z}, l, \theta)}}[m, n]}
$$

where $R_{O_{(\mathcal{Z}, l, \theta)}}[m, n]$ and $R_{i_{(\mathcal{Z}, l, \theta)}}[m, n]$ are, respectively, the masking response, for a referenced and a distorted coefficient, taken from Equation (2). $\mathcal{Z} \in\{\Theta, \Upsilon\}$, denotes the orientation and local responses, respectively. Equation (9) provides a measurement of the depreciation of the response energy over both the intra-frequency and inter-orientation channels.

The last stage gathers the set of distortion measures, $\mathcal{D}_{\mathcal{T}(l, \theta, m, n)}$, the set of percentage responses, $\mathcal{R}_{\mathcal{P}(l, \theta, m, n)}$ and performs visually adaptive coefficient pruning. By comparing $\mathcal{D}_{\mathcal{T}(l, \theta, m, n)}$ and $\mathcal{R}_{\mathcal{P}(l, \theta, m, n)}$ to a set of pre-determined JNND thresholds, $\mathcal{T}_{\mathcal{D}}$ and $\mathcal{T}_{\mathcal{P}}$, respectively, a coefficient is truncated (Equation 8) to a perceptually optimal bit-plane level, $i_{o p t}$, only when a distortion measure from $\mathcal{D}_{\mathcal{T}}(l, \theta, m, n)$ is less than or equal to a JNND threshold, $\mathcal{T}_{\mathcal{D}(l, \theta)}$ and when a percentage response from $\mathcal{R}_{\mathcal{P}(l, \theta, m, n)}$ is less than or equal to a percentage response threshold $\mathcal{T}_{\mathcal{P}(l, \theta)}$. Thus,

$$
\tilde{\mathbf{X}}_{i_{o p t l}, \theta}[m, n]=\mathbf{X}_{l, \theta}[m, n] \times \frac{\left(P-i_{o p t}\right)}{P}
$$

where

$$
\begin{array}{r}
i_{\text {opt }}=\max \left\{i \in \mathcal{B} \mid\left(\mathcal{D}_{\left.\mathcal{T}_{i(l, \theta, m, n)} \leq \mathcal{T}_{\mathcal{D}(l, \theta)}\right)}\right.\right. \\
\left.A N D\left(\mathcal{R}_{\mathcal{P} i(l, \theta, m, n)} \leq \mathcal{T}_{\mathcal{P}(l, \theta)}\right)\right\}
\end{array}
$$

B. Determining $\mathcal{T}_{\mathcal{D}(l, \theta)}$ and $\mathcal{T}_{\mathcal{P}(l, \theta)}$

Both $\mathcal{T}_{\mathcal{D}(l, \theta)}$ and $\mathcal{T}_{\mathcal{P}(l, \theta)}$ were derived from subjective experiments. For each orientation $\left(\theta_{\alpha}\right)$ and each frequency level $(l)$,there is a set of pre-determined thresholds $\mathcal{T}_{\mathcal{D}}$ and $\mathcal{T}_{\mathcal{P}}$, for $\theta=\{1,2,3\}$ and $l=\{1,2,3,4,5\}$. These thresholds have been obtained through the testing of approximately 2560 (32x32 pixels) 16-bit medical greyscale sub-images, for each modality. These sub-images originated from a particular base image (512x512 pixels), which was distorted in 10 different ways through bit-plane filtering. Each of these 10 distorted images were then partitioned into 256 (32x32 pixels) individual pieces. Sub-image testing is preferred in this case over the complete image testing because it is able to quantify the 
different local thresholds levels in different regions within images, i.e., the segmented test is better equipped to capture the localised variation in image quality. The threshold level for the experiment is set at the JNND level for visually lossless quality encoding. Once the JNND level of test materials have been mapped, the thresholds $\mathcal{T}_{\mathcal{D}}$ and $\mathcal{T}_{\mathcal{P}}$ can be determined by soliciting the responses (2) and (7) of the sub-images in the JNND map. In other words, only sub-images at the JNND level will be used to determined the thresholds $\mathcal{T}_{\mathcal{D}(l, \theta)}$ and $\mathcal{T}_{\mathcal{P}(l, \theta)}$. Finally, fine tuning was performed through a stringent test of flipping back and forth the encoded image with the original image. This employs the temporal sensitivities of the HVS to ensure that 'distortion flickers' between the two images are not perceivable.

\section{JPEG 2000 Adaptation}

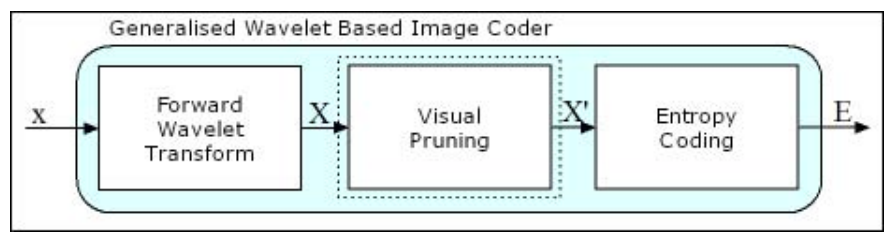

Fig. 4. A generalised Wavelet-based image coder embedded with the Visual Pruning algorithm. The Visual Pruning algorithm is applied immediately after a forward transform. Note that the Quantiser was disabled (step size set to 1).

The VP algorithm is independent and does not require a specialised decoder. Thus it can be implemented in any Wavelet based coding framework (Figure 4). Here, the VP algorithm is applied to the wavelet coefficients immediately after a forward Wavelet transform. The alternative is to apply the algorithm on a per code block basis, however, this method can significantly impede the accuracy of the distortion metric thus leading to lower coding performance [27].

\section{Performance Evaluation}

The proposed coder (PC-PCT) in this paper will be evaluated against four benchmark coders. The first is the LOCO lossless coder [28], the second is the NLOCO near-lossless coder [28], the third is the JPEG2000 lossless mode (J2KL) [24] and the fourth is a variant of the (PC-PCT), (PC-BCT) [7], which employs a bit-plane coefficient truncation (BCT) approach. A parameter, $d$, for NLOCO is set to 2 . Here, $d$ represents the maximum pixel difference between the original image and the compressed image. Current results (Table I \& II), in terms of bits-per-pixel(bpp), show that the coding performance of the (PC-PCT) outperforms the LOCO and J2KL lossless coders in all instances, averaging $70 \%$ more compression. On the other hand, there was on average, a 5\% and $14 \%$ compression gain over the NLOCO near-lossless coder and the PC-BCT coder, respectively. A PCT approach provides a more accurate account of the visual distortions since each coefficient is pruned at finer fractional steps depending on the precision $P$, whereas, in the BCT approach each coefficient is pruned at fixed step sizes in powers of $2\left(1,2,4, \ldots, 2^{31}\right)$. A drawback of the PCT approach is that it has a higher computational complexity over the BCT when $P$ is greater than 32 , that is, the greater the precision the greater the computational complexity. The impact of $P$ on the coding performance is dependant on the threshold values $\mathcal{T}_{\mathcal{D}(l, \theta)}$ and $\mathcal{T}_{\mathcal{P}(l, \theta)}$. Generally, it is desirable to use larger values of $P$ when determining the aforemention JNND visual thresholds. Nevertheless and more importantly, no distortions were perceivable in the images compressed by PC-PCT (Figure 5).

\begin{tabular}{|l|c|c|c|c|c|}
\hline Image & Dimensions & Image & \multicolumn{3}{|c|}{ Bitrate (bpp) } \\
\cline { 4 - 6 } Name & pixels) & Type & LOCO & J2 KL & PC- PCT \\
\hline \hline Ankle 1 & $2572 \times 2040$ & CR & 5.852 & 5.892 & 3.425 \\
\hline Ankle 2 & $1516 \times 2044$ & CR & 5.695 & 5.707 & 3.201 \\
\hline Body 1 & $512 \times 512$ & CT & 4.191 & 4.327 & 2.136 \\
\hline Body 2 & $512 \times 512$ & CT & 5.933 & 6.235 & 4.047 \\
\hline Body 3 & $512 \times 512$ & CT & 6.851 & 6.987 & 4.007 \\
\hline Brain 1 & $512 \times 512$ & CT & 3.380 & 3.558 & 1.768 \\
\hline Brain 2 & $512 \times 512$ & CT & 3.841 & 3.500 & 1.304 \\
\hline Brain 3 & $512 \times 512$ & MR & 4.687 & 4.650 & 3.341 \\
\hline Brain 4 & $208 \times 256$ & MR & 3.898 & 1.523 & 3.749 \\
\hline Brain 5 & $512 \times 512$ & CT & 3.681 & 3.789 & 1.857 \\
\hline Brain 6 & $512 \times 512$ & CT & 3.597 & 3.230 & 1.144 \\
\hline Brain 7 & $512 \times 512$ & CT & 6.175 & 6.459 & 4.237 \\
\hline Brain 8 & $512 \times 512$ & CT & 3.962 & 3.627 & 1.346 \\
\hline Brain 9 & $512 \times 512$ & CT & 3.190 & 2.815 & 0.957 \\
\hline Chest 1 & $2496 \times 2048$ & CR & 6.786 & 6.847 & 4.683 \\
\hline Chest 2 & $2496 \times 2048$ & CR & 6.821 & 6.874 & 4.711 \\
\hline Chest 3 & $2496 \times 2048$ & CR & 6.259 & 6.312 & 4.108 \\
\hline Chest 4 & $2496 \times 2048$ & CR & 6.198 & 6.288 & 4.079 \\
\hline Chest 5 & $2496 \times 2048$ & CR & 6.020 & 6.051 & 3.788 \\
\hline Chest 6 & $1516 \times 2044$ & CR & 5.912 & 5.965 & 3.526 \\
\hline Elbow & $2044 \times 1514$ & CR & 6.177 & 6.200 & 3.835 \\
\hline Knee & $512 \times 512$ & MR & 7.143 & 6.903 & 5.211 \\
\hline Leg & $2040 \times 2570$ & CR & 5.759 & 5.740 & 3.234 \\
\hline Liver & $512 \times 512$ & CT & 5.029 & 4.942 & 1.951 \\
\hline Neck & $2040 \times 2570$ & CR & 4.674 & 4.598 & 2.636 \\
\hline Pelvis & $3732 \times 3062$ & CR & 5.915 & 5.993 & 3.693 \\
\hline SideBrain & $256 \times 256$ & MR & 5.972 & 5.927 & 4.071 \\
\hline Spine 1 & $2040 \times 2570$ & CR & 6.437 & 6.482 & 4.155 \\
\hline Spine 2 & $512 \times 512$ & CT & 3.526 & 3.458 & 1.664 \\
\hline Spine 3 & $512 \times 512$ & CT & 6.106 & 6.375 & 4.290 \\
\hline & AVERAGE & & 5.322 & 5.316 & 3.132 \\
\hline
\end{tabular}

TABLE I

SHOWS THE CODING PERFORMANCE, IN TERMS OF BITRATE, BETWEEN THE PC-PCT AND THE LOSSLESS CODERS, LOCO AND J2KL. EACH IMAGE HAS A MAXIMUM BIT-DEPTH OF 16 BITS PER PIXEL.

\section{Conclusion}

This paper presents an improved visual pruning algorithm for perceptually lossless image coding. Built on the JPEG2000 coding framework [24] and embedded with an advanced $\mathrm{Hu}-$ man vision model, the VP algorithm can identify and remove visually insignificant/irrelevant information. In terms of coding performance, the PC outperforms its lossless counterparts in all instances. The key features of the VP algorithm is its simplicity and modularity. Hence, it does not require a specialised decoder and can be implemented into any wavelet based image coder while maintaining bit-stream compliance. More importantly, there was no perceivable loss in fidelity. 


\begin{tabular}{|l|c|c|c|c|c|}
\hline Image & Dimensions & Image & \multicolumn{3}{|c|}{ Bitrate (bpp) } \\
\cline { 4 - 6 } Name & (pixels) & Type & PC-BCT & NLOCO & PC-PCT \\
\hline \hline Ankle 1 & $2572 \times 2040$ & CR & 3.945 & 3.557 & 3.425 \\
\hline Ankle 2 & $1516 \times 2044$ & CR & 3.758 & 3.395 & 3.201 \\
\hline Body 1 & $512 \times 512$ & CT & 2.650 & 2.494 & 2.136 \\
\hline Body 2 & $512 \times 512$ & CT & 4.475 & 4.116 & 4.047 \\
\hline Body 3 & $512 \times 512$ & CT & 4.675 & 4.517 & 4.007 \\
\hline Brain 1 & $512 \times 512$ & CT & 2.040 & 1.963 & 1.768 \\
\hline Brain 2 & $512 \times 512$ & CT & 1.595 & 2.058 & 1.304 \\
\hline Brain 3 & $512 \times 512$ & MR & 3.981 & 2.959 & 3.341 \\
\hline Brain 4 & $208 \times 256$ & MR & 2.500 & 1.624 & 3.749 \\
\hline Brain 5 & $512 \times 512$ & CT & 2.163 & 2.165 & 1.857 \\
\hline Brain 6 & $512 \times 512$ & CT & 1.427 & 1.893 & 1.144 \\
\hline Brain 7 & $512 \times 512$ & CT & 4.641 & 4.339 & 4.237 \\
\hline Brain 8 & $512 \times 512$ & CT & 1.640 & 2.124 & 1.346 \\
\hline Brain 9 & $512 \times 512$ & CT & 1.213 & 1.616 & 0.957 \\
\hline Chest 1 & $2496 \times 2048$ & CR & 4.803 & 4.468 & 4.683 \\
\hline Chest 2 & $2496 \times 2048$ & CR & 4.818 & 4.506 & 4.711 \\
\hline Chest 3 & $2496 \times 2048$ & CR & 4.320 & 3.948 & 4.108 \\
\hline Chest 4 & $2496 \times 2048$ & CR & 4.288 & 3.894 & 4.079 \\
\hline Chest 5 & $2496 \times 2048$ & CR & 4.061 & 3.737 & 3.788 \\
\hline Chest 6 & $1516 \times 2044$ & CR & 4.015 & 3.598 & 3.526 \\
\hline Elbow & $2044 \times 1514$ & CR & 4.253 & 3.868 & 3.835 \\
\hline Knee & $512 \times 512$ & MR & 5.897 & 4.838 & 5.211 \\
\hline Leg & $2040 \times 2570$ & CR & 3.789 & 3.422 & 3.234 \\
\hline Liver & $512 \times 512$ & CT & 2.690 & 2.783 & 1.951 \\
\hline Neck & $2040 \times 2570$ & CR & 3.042 & 2.779 & 2.636 \\
\hline Pelvis & $3732 \times 3062$ & CR & 4.093 & 3.683 & 3.693 \\
\hline SideBrain & $256 \times 256$ & MR & 4.996 & 3.745 & 4.071 \\
\hline Spine 1 & $2040 \times 2570$ & CR & 4.498 & 4.173 & 4.155 \\
\hline Spine 2 & $512 \times 512$ & CT & 2.620 & 1.888 & 1.664 \\
\hline Spine 3 & $512 \times 512$ & CT & 4.675 & 4.255 & 4.290 \\
\hline & & 3.585 & 3.280 & 3.132 \\
\hline
\end{tabular}

TABLE II

SHOWS THE CODING PERFORMANCE, IN TERMS OF BITRATE, BETWEEN THE PC-PCT,PC-BCT AND NLOCO WITH $d=2$. EACH IMAGE HAS A MAXIMUM BIT-DEPTH OF 16 BITS PER PIXEL.

\section{ACKNOWLEDGMENT}

The authors would like to thank Southern Health Monash Medical Centre and the patients for permission to use their images.

\section{REFERENCES}

[1] M. Krol, "Telemedicine," IEEE Potentials, vol. 16, pp. 29-31, Oct-Nov 1997.

[2] B. J. Erickson, "Irreversible Compression Of Medical Images," Society For Computer Applications In Radiology White Paper, pp. 1-9, November 2000 .

[3] T. Ishigaki, S. Sakuma, M. Ikeda, Y. Itoh, M. Suzuki, and S. Iwai, "Clinical Evaluation of Irreversible Image Compression: Analysis of Chest Imaging with Computed Radiography," Journal of Radiology, vol. 175 , pp. $739-743,1990$.

[4] A. J. Maeder and M. Deriche, "Establishing perceptual limits for medical image compression," Proceedings SPIE, Medical Imaging 2001: Image Perception and Performance, vol. 4324, pp. 204 - 210, 2001.

[5] R. M. Slone, D. H. Foos, B. R. Whiting, E. Muka, D. A. Rubin, T. K. Pilgram, K. S. Kohm, S. S. Young, P. Ho, and D. D. Hendrickson, "Assessment of Visually Lossless Irreversible Image Compression: Comparison of Three Methods by Using an Image-Comparison Workstation," Radiology - Computer Applications, vol. 215, no. 2, pp. 543 - 553, 2000.

[6] M. Sung, H. Kim, E. Kim, J. Kwak, J. Yoo, and H. Yoo, "Clinical Evaluation of JPEG2000 Compression for Digital Mammography," IEEE Transaction On Nuclear Science, vol. 49, no. 3, pp. 827 - 832, 2002.
[7] D. Wu, D. M. Tan, M. Baird, J. DeCampo, C. White, and H. R. Wu, "Performance evaluation of a perceptually lossless medical image coder," in Proceedings of 2004 International Symposium on Intelligent Multimedia, Video \& Speech Processing, Hong Kong, October 2004, pp. 366-369.

[8] D. Taubman, "High Performance scalable image compression with EBCOT," IEEE Transaction on Image Processing, vol. 9, no. 7, pp. $1151-1170,2000$.

[9] D. M. Tan, H. R. Wu, and Z. Yu, "Perceptual Coding Of Digital Monochrome Images," IEEE Signal Processing Letters, vol. 11, no. 2, pp. $239-242,2004$.

[10] D. Wu, D. M. Tan, and H. R. Wu, "Visually Lossless Adaptive Compression Of Medical Images," Proc. of The Fourth International Conference on Information, Communications \& Signal Processing and Fourth Pacific Rim Conference on Multimedia, pp. 458 - 463, 2003.

[11] _ " "Vision Model Based Approach To Medical Image Compression," Proc. of the International Symposium on Consumer Electronics, 2003, Paper No.: ISCE3058.

[12] A. Said and W. A. Pearlman, "A New Fast and Efficient Image Codec Based on Set Partitioning in Hierarchical Trees," IEEE Transaction on Circuits and Systems for Video Technology, vol. 6, no. 1, pp. 243 - 250, June 1996.

[13] R. C. Gonzalez and R. E. Woods, Digital Image Processing, 2nd ed. Prentice Hall, Inc., 2002.

[14] B. Girod, "What's Wrong with Mean-squared Error?" Digital Images and Human Vision, pp. 207-220, 1993.

[15] Z. Yu, H. R. Wu, S. Winkler, and T. Chen, "Vision Model Based Impairment Metric To Evaluate Blocking Artifacts in Digital Video," Proceedings of the IEEE, vol. 90, no. 1, pp. 154-169, Jan 2002.

[16] P. C. Teo and D. J. Heeger, "Perceptual Image Distortion," Proceedings of SPIE, no. 2179, pp. 127 - 141, 1994.

[17] — , "Perceptual Image Distortion," In Proc. of IEEE Int. Conf. on Image Processing, vol. 2, pp. 982 - 986, Nov. 1994.

[18] A. B. Watson and J. A. Solomon, "A Model of Visual Contrast Gain Control and Pattern Masking," Journal of the Optical Society of America A, pp. 2379 - 2391, 1997.

[19] B. Wandell, Foundations of Vision. Sinauer Associates, Inc., 1995.

[20] S. Mallat, "Wavelets for a Vision," Proceedings of the IEEE, vol. 84, no. 4, pp. $604-614$, apr 1996.

[21] E. P. Simoncelli, W. T. Freeman, E. H. Adelson, and D. J. Heeger, "Shiftable Multiscale Transform," IEEE Trans. on Information Theory, vol. 38, no. 2, pp. 587-607, 1992.

[22] S. G. Mallat, "A Theory for Multiresolution Signal Decomposition: The Wavelet Representation," IEEE Trans. on Pattern Analysis and Machine Intelligence, vol. 11, no. 7, pp. 674 - 693, July 1989.

[23] M. Antonini, M. Barlaud, P. Mathieu, and I. Daubechies, "Image Coding Using Wavelet Transform," IEEE Transaction on Image Processing, vol. 1, no. 2, pp. 205-220, Apr. 1992.

[24] D. Taubman and M. W. Marcellin, JPEG2000: Image compression fundamentals, standard and practice, 1st ed. Kluwer Academic Plublishers, 2002.

[25] ACR-NEMA, "DICOM Standard," 2004. [Online]. Available: http: //medical.nema.org/

[26] R. Safranek and J. Johnston, "A Perceptually Tuned Sub-band Image Coder with Image Dependent Quantization and Post-quantization Data Compression," Proc. of IEEE ICASSP, pp. 1945-1948, 1989.

[27] D. Wu, D. M. Tan, and H. R. Wu, "Visually Lossless Adaptive Compression For Medical Images With JPEG 2000," Proc. of the International Symposium on Consumer Electronics, pp. 96-100, 2004.

[28] M. Weinberger, G. Seroussi, and G. Sapiro, "The LOCO-I Lossless Image Compression Algorithm: Principles and Standardization into JPEG-LS," IEEE Transaction on Image Processing, vol. 9, pp. 13091324, August 2000, Hewlett-Packard Laboratories Technical Report No. HPL-98-193R1, November 1998, revised October 1999. 

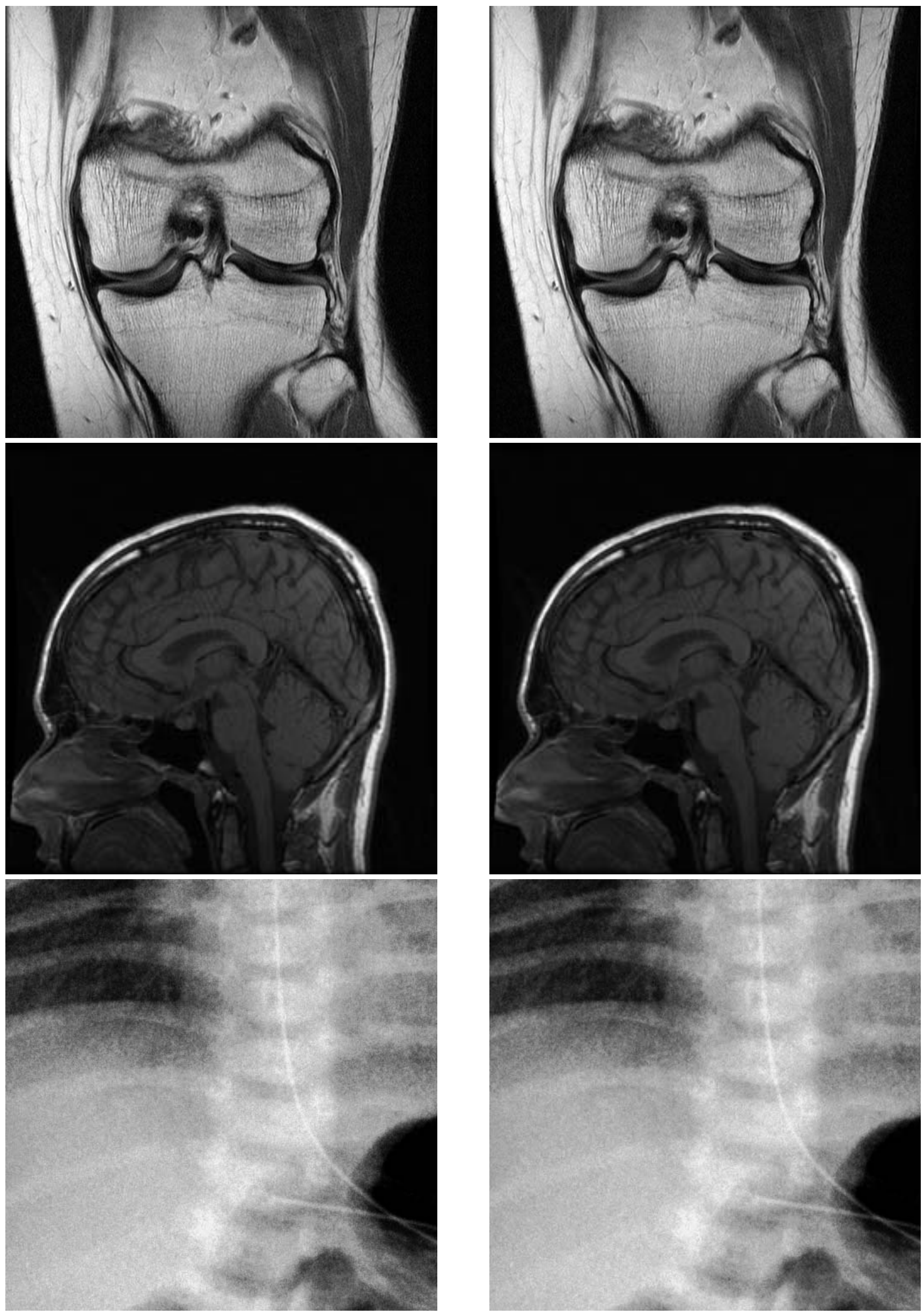

Fig. 5. Left: Original. Right: Proposed Coder. Top down: Knee; SideBrain; Chest6(cropped). 